\title{
Program Realization and Analysis of Periodic Function Application
}

\author{
YANG Dongchen ${ }^{1, a}$, SHI Duo ${ }^{1, a}$, XURIGAN Saiyin ${ }^{1, a}$, HU Chenna ${ }^{1, a}$, LIU Yi*1,a \\ ${ }^{1}$ Beijing Institute of Fashion Technology, Chaoyang District, Beijing, China \\ amableliuyi@163.com
}

Keywords: Periodic Function; Simulation; Program

\begin{abstract}
The dissertation briefly expounded the definition and mathematical analysis of a periodic function. Described the program development process of simulating periodic function curve and associated knowledge by computer language. It also given in detail the principle of production and flow chart. Introduced the function and practical application.
\end{abstract}

\section{Preface}

The use of periodic function is prevalent, it can be used in many fields including science of mathematics, financial periodic economic fluctuation, daily variation regularity of body temperature in medicine and so on. In the PE, it also played a important role. Because of its abstract character, there are many difficulties in understanding its application and studying. Therefor, according to the principle and character of the periodic function, realizing a program with the teaching function of simulation, automatic analysis, making an example at any time by computer language.

The definition of function's periodicity : As a function, if there is a nonzero constant $\mathrm{T}$ making $\mathrm{x}$ true when take any value within the domain. So we call the function $\mathrm{y}=\mathrm{f}(\mathrm{x})$ periodic function, and $\mathrm{T}$ was called period of the function.

\section{Design of periodic function waveform program}

Considering the rich class provided by the MFC and efficient compilation environment of VC, Here adapt the object-oriented method based on $\mathrm{c}++$ language to develop in the environment of VC MFC framework.

The program of design and relies of periodic function need three components including the acquisition of function data, data processing and function graphic output.

The thought of design is that users can chose a periodic function already given by designer, and type in the required parameters in a given dialog box to determine the function characteristics, then, the computer will generate a series of associated data point $(\mathrm{x}, \mathrm{y})$ by data processing components and output component will get corresponding data by a certain algorithm. Thus user can get periodic function graph.

Function data acquisition components need collect information including the function and its parameters. The given form restrict user to type the certain parameter format. For example, the sine function format is:

$$
y=a * \sin (b * x+c)
$$

The given format make the programmer send parameters to data processing component.

Data processing component invoke internal functions based on the function user have chosen. Acquisition of function data component send the periodic function and relevant parameter to data processing, after loop computation, there will be the number of data points, and graphic output component will display diagram.

Function graphic output, through function calls in the CDC class, component set up and draw two main part of the screen: the design of GDI, including design of the canvas, transverse and longitudinal axis, drawing of the grid lines and so on, the design of function graphic.The data relevance among the three components in the program data and the relevance with Input/output devices. 


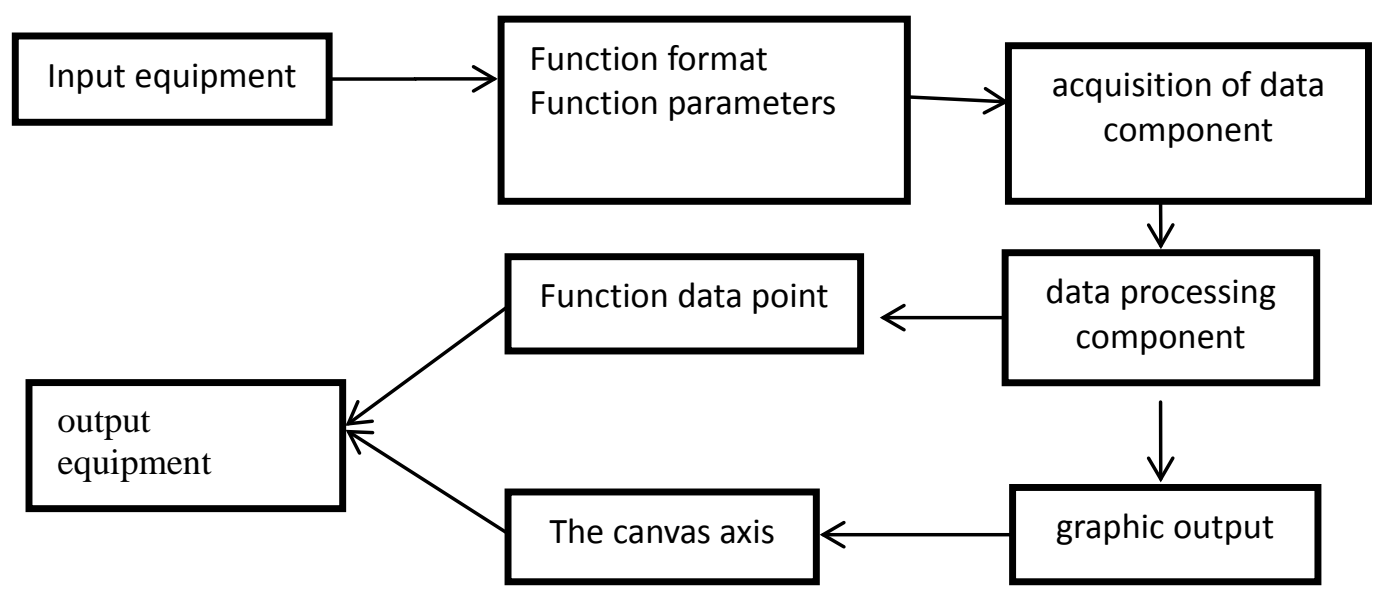

Fig1. The relationship between components and IO equipments relationship

\section{The realization of periodic functional program}

\section{The functional components to collect data}

The function of the components is to input information into the designed window, then pass them to the special components processing the data, and the information which the components collect.

1.Choose the function wanted

Throng building a series of menus and dialog in MFC single document to realize the functions, and the specific process is as following:

Firstly, add some drop-down menu button in the single document. Secondly, choose the Periodic function such as sine, cosine and so on in the drop-down menu box, as well as set the functional parameters. This take sine function as an example to design the distance. In order to identify the format of function, link base class, CPeroidFunctionBasic10View ,to the menus of sine function, and add private variable, $\mathrm{m} \_n$ FunctionStyle, to base class, CPeroidFunctionBasic10View, making its initial value set to 0 and identifying the options, $\mathrm{m} \_$nFunctionStyle $=1$, in the means of onsin().

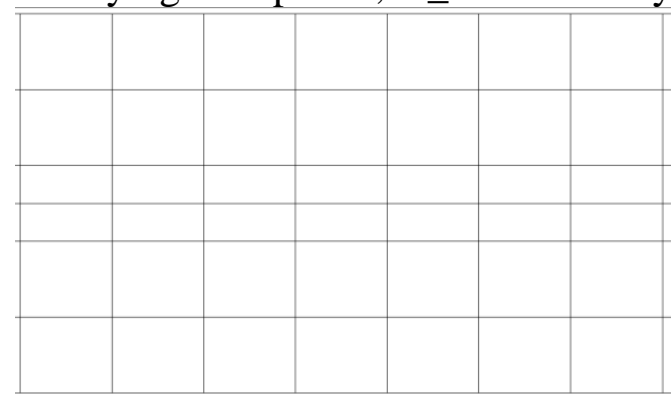

Fig2.The results of function selection process

2.The selected function customization parameters input

Add a related events, OnParameterset(), which base class is CPeroidFunctionBasic10View to the settings menu item of function parameter, the OnParameterset() events need to invoke a dialog box to achieve the input of parameters, the dialog box in this case shown below .Relate the dialog box to class, SettingDlg, at the same time respectively associated three EditControl module to variate m_nParameterA, m_nParameterB, m_nParameterC one-to-one and add three the same variable into the CPeroidFunctionBasic10View class .To achieve the introduction of date ,till now, the design of the component to collect data of function finished. 


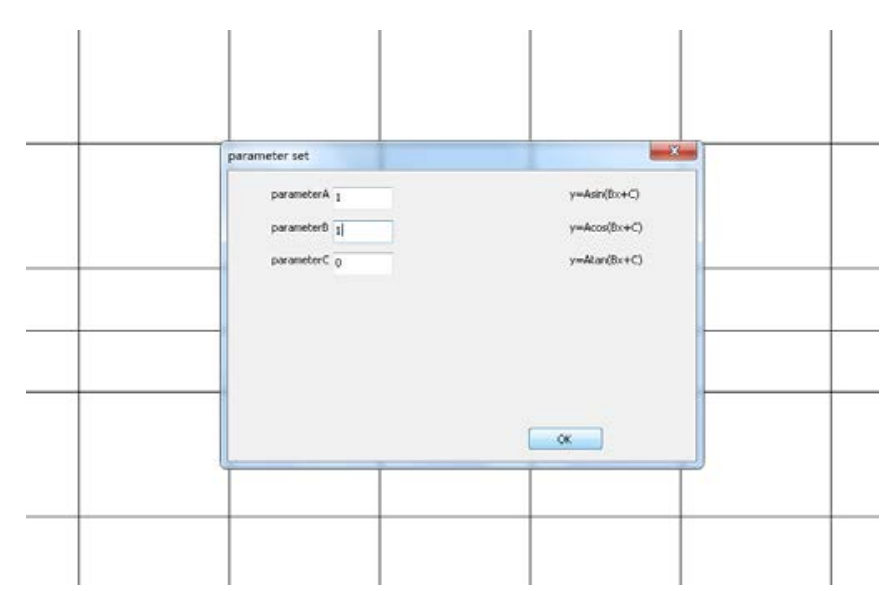

Fig3. Schematic diagram of self-defined parameter input

\section{The functional components to calculation processing data}

The design of the functional components to calculation processing data is relatively simple, double-click the plotting area after choose the type of periodic function and setting its parameters, the goal of it is to achieve data processing and generate a series of reference points $(i, j)$.

Add dblclick OnLButtonDblClk(UINT nFlags, CPoint point), to CPeroidFunctionBasic10View class . The function which OnLButtonDblClk method achieved in the functional components to processing data has two parts .A part of it is to make sure the choose of function style and receive parameters ,the another is the calculation of points .Of which, the choose of function style is confirmed by switch .

While directly write three parameters of double $y$ $=\mathrm{m} \_n P a r a m e t e r A * \sin \left(m \_n P a r a m e t e r B * x+m \_n P a r a m e t e r C\right)$ into $y$ to complete the expression. As well as, the generation of $\mathbf{i}, \mathbf{j}$ relates to function's drawing and Settings, it will be narrated in next section.

\section{The functional components to output function's graph}

The functional components can come true two function, it is canvas' setting and functional image's drawing.

Due to the Pixel do not have decimals, if you want to guarantee functional image coherent, distinct and sparse ,you need to calculate the date as more as possible to trace and connect. Obviously ,in the process of tracing point, if we let all the coordinates in $\mathrm{X}$ axis to be integer ,it will cause function graph too many distortions .So we should set canvas as big as possible ,and relate the chosen points to the integral pixel in the screen by shrinking canvas several times.Here, we adopt the method that shrink i, abscissa of the point in X axis,100 times, then we can get $\mathrm{X}$ and through calculating to get $\mathrm{Y}$. What's more, we can get $\mathrm{j}$, ordinate of practical pixel ,by certain conversion and algorithm to Y. Now we use library function of CClientDC class to portray, and set window to be virtual pixel window, dc.SetWindowExt(1800,10000). At the same time ,set paintbrush to be CPen pen(PS_SOLID,1,RGB(255,0,0)).

As we all know, if we set origin of coordinates in the top left corner of the screen, the positive direction of $\mathrm{X}$ axis is right, the positive direction of $\mathrm{Y}$ axis is downward. So when tracing point by LINETO function, we need to transform $(\mathrm{x}, \mathrm{y})$ to point $(\mathrm{i}, \mathrm{j})$ which is related to the screen .The Implementation approach is $\mathrm{X}$, shrinking each i point 100 times, calculated by sine function .So we get the relationship between $\mathrm{Y}$ and $\mathrm{J}$ is

$\mathrm{j}=5000-(1000 * \mathrm{y})$

Then we get the pixel $(i, j)$ whose abscissa is $J$. 


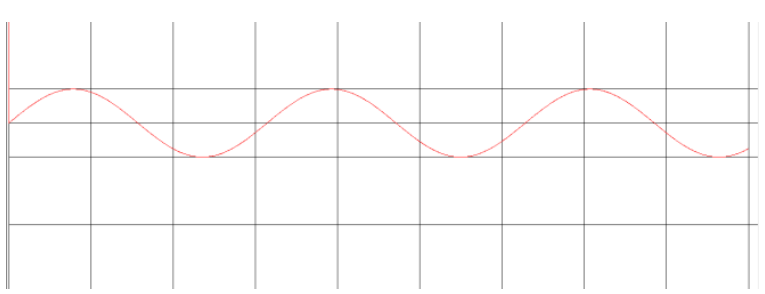

Fig4. Schematic diagram of function's output results

\section{Application of periodic function in practice}

It is convenient to research and study the periodic function by using the program of periodic function curve fitting, at the same time, some areas can also be better to collect the study data by simulating function graph. For example the teaching, traditional teaching is mainly conducted through the blackboard writing and oral, as the result, it is difficult for leaners to understand the abstract concept like cosine wave real-time status in physics. If the dynamic demonstration can be made by program, the students can intuitively analyze the state related to the physical of the fluctuations in a single point at a particular time and have a more intuitive observation and data analysis about continuous state of motion. Observing the change of data and wave contributes to the teaching by altering range and period. This program meet the needs of simulating dynamic demonstration and static analysis of all functions. Besides, it can speed up the students to understand and short the teaching class by changing the function data. The program meet the requirement of the data study about the simulation demonstration of periodic function such as square wave, sawtooth wave. In general, program play an important role in the study and research on periodic function.

\section{Acknowledgements}

This work was financially supported by Beijing College Student Research Training Program (2014,2015), Open Project of Digital and Interactive Media Key Laboratory (KF2013-01, KF2013-13), Beijing Institute of Fashion Technology Teaching reform and innovation team project

(JGTD-1404) , PHR(IHLB)-Innovative Research Team PTTBIFT_TD_002, Beijing education reform project (2013-ms145)

\section{References}

[1]Jin Liang, Research on Teaching of Periodic Function Conception, Science Examination Research, Oct,2014

[2]Yanping Wang, Zonghan $\mathrm{Xi}$, Research on Computer Fitting Periodic Function and Its Application, Jisuanji Yu Xiandaihua, May, 2007

[3] Stanley B. Lippman, Josée Lajoie, Barbara E. Moo. C++ Primer Publishing House Of Electronics Industry

[4] Stephen Prata. C++ Primer Plus[M] Publishing House Of Electronics Industry. 